\title{
Incidence and prevalence of moyamoya disease in urban China: a nationwide retrospective cohort study
}

\author{
Yixin Sun (10 , ${ }^{1}$ Guoyu Zhou, ${ }^{2}$ Jingnan Feng, ${ }^{1}$ Lu Chen, ${ }^{3}$ Guozhen Liu, ${ }^{4}$ Jinxi Wang, ${ }^{5}$ \\ Qingliang Wang, ${ }^{6}$ Junyou Yu, ${ }^{1}$ Xiwang Yang, ${ }^{1}$ Zheng Yang, ${ }^{1}$ Pei Gao, ${ }^{1}$ \\ Shengfeng Wang, ${ }^{1}$ Siyan Zhan ${ }^{1}$
}

To cite: Sun Y, Zhou G, Feng J, et al. Incidence and prevalence of moyamoya disease in urban China: a nationwide retrospective cohort study. Stroke \& Vascular Neurology 2021;0. doi:10.1136/svn-2021000909

\section{- Additional online} supplemental material is published online only. To view, please visit the journal online (http://dx.doi.org/10.1136/svn2021-000909).

YS and GZ contributed equally.

Received 30 January 2021 Revised 23 March 2021 Accepted 14 April 2021
Check for updates

(c) Author(s) (or their employer(s)) 2021. Re-use permitted under CC BY-NC. No commercial re-use. See rights and permissions. Published by BMJ.

For numbered affiliations see end of article.

\section{Correspondence to} Professor Siyan Zhan; Siyan-zhan@bjmu.edu.cn

Professor Shengfeng Wang; shengfeng1984@126.com

\section{ABSTRACT}

Background and objective Moyamoya disease (MMD) is an increasingly recognised cause of stroke, mainly described in East Asia. China is the largest nation in Asia, but few studies reported the epidemiology of MMD, especially at a national level. We aimed to estimate the incidence and prevalence of MMD in China.

Methods We performed a population-based study using data from the national databases of Urban Basic Medical Insurance between 2013 and 2016, covering approximately 0.50 billion individuals. MMD cases were identified by diagnostic code (International Classification of Diseases, 10th Revision I67.5) or related diagnostic text.

Results A total of 1987 MMD patients (mean age $44.45 \pm 14.30$ years, female-to-male ratio 1.12) were identified, representing a national crude incidence of 0.59 (95\% Cl: 0.49 to 0.68$)$ and a prevalence of 1.01 (95\% Cl: 0.81 to 1.21 ) per 100000 person-years in 2016. Rates were higher in females than in males for the incidence ( 0.66 vs 0.52 ) and prevalence ( 1.05 vs 0.90 ). And the age-specific rates showed a bimodal distribution, with the highest peak in middle-aged group and the second peak in child group.

Conclusions Our results confirm that MMD is relatively common in East Asians, but the rates in China were lower than those in other East Asian countries such as Japan and Korea. The unique epidemiological features, including a relatively weak female predominance and a shift in the highest peak of incidence from children to adults, revealed new sight into MMD. Further research is expected to explore the potential pathogenesis of MMD.

\section{INTRODUCTION}

Moyamoya disease (MMD) is a rare cerebrovascular disease of unknown pathogenesis. It is characterised by progressive stenosis or occlusion of the intracranial portion of the internal carotid artery and its proximal branches. ${ }^{1}$ Patients with MMD often develop severe ischaemic or haemorrhagic events, including transient ischaemic attack, stroke, intracranial haemorrhage, seizures or cognitive impairment, which result in a high rate of disability and even death. ${ }^{23}$

MMD was first described in Japan in 1957, and subsequently, it has been observed in people of different ethnic backgrounds throughout the world. However, the epidemiological features showed wide regional variations. ${ }^{4}$ MMD was relatively common in East Asian countries such as Japan ${ }^{5-8}$ and South Korea, ${ }^{9}{ }^{10}$ while it was rarely discovered in the United States ${ }^{11} 12$ and Europe. ${ }^{13} 14$ The incidence was $0.94-1.13$ per 100000 people and the prevalence was $5.22-10.50$ per 100 000 people in Japan, and the rates were 1.7-4.3 and 6.5-18.1 per 100000 personyears in South Korea, respectively. ${ }^{7-10}$ China is the largest nation in East Asia, but data on MMD were scarce and inconsistent. One population-based study from Taiwan reported an annual incidence of 0.15 per 100000 person-years from 2000 to 2011, far below that of Japan and South Korea. ${ }^{15}$ Meanwhile, only three hospital-based studies were conducted in mainland China. ${ }^{16-19}$ These existing studies were performed about 10 years ago or were limited in a single centre or selected hospitals. Additionally, no further studies were available to evaluate the rates among different sex and age groups and the disease burden such as costs and length of stay associated with MMD in mainland China. These data could provide clues to understand the pathogenesis and inform policy-makers for the management of MMD.

Therefore, this nationally population-based study aimed to estimate the incidence and prevalence of MMD and their patterns across sex, age and geographical region, as well as to investigate the hospital charges and length of stay associated with MMD in mainland China.

\section{METHODS}

\section{Data sources}

The Chinese government has implemented two nationwide universal health insurance schemes in urban areas: the Urban Employee Basic Medical Insurance (UEBMI) aimed at urban working and retired employees (ie, 
employers and employees from government agencies and institutions, state-owned enterprises, private businesses, social organisations, and other private entities) and the Urban Residence Basic Medical Insurance (URBMI) for urban residents without formal employment (ie, children, students and unemployed citizens) ${ }^{20}$ By 2016, these two programmes covered more than $95 \%$ of the entire population in urban areas. ${ }^{21}$ All medical records of the insured population were kept in the database of their registered province, regardless of where they receive medical services (seeking medical treatment outside their residential location) or the proportion they pay for medical services (even no medical expense is reimbursed).

We obtained data from the UEBMI and URBMI database. The claims database contained information about enrollees' demographic characteristics (province, date of birth, sex, etc), insured information (date of enrollment and withdrawal, insurance type, etc), inpatient and outpatient medical records (date of medical service, diagnostic text, diagnostic code, etc) and medical costs.

\section{Study population}

Claims data covered a national population of approximately 0.50 billion people in 22 provinces from 1 January 2013 to 31 December 2016. We excluded nine provinces (Fujian, Tibet, Sichuan, Beijing, Shanghai, Tianjin, Ningxia, Hebei and Jiangxi) due to the absence or abnormality of crucial information (eg, primary diagnosis), covering only one type of insurance, or reporting policy exemptions. All claim records were anonymised for study purposes.

\section{Case identification}

Patients with MMD were identified by diagnostic code or diagnostic text, based on International Classification of Diseases, 10th Revision code I67.5, ${ }^{12}{ }^{14}$ and medical terms in Chinese and English including MMD, spontaneous occlusion of the circle of Willis, abnormal vascular network at the base of the brain, moyamoya vessels and MMD. To avoid missing patients, we constructed a relatively loose algorithm using fuzzy string matching to extract all potential MMD patients in the database. Then two neurologists reviewed the diagnosis of each potential patient independently to ascertain the definite MMD patients. Patients were excluded if (1) they had been diagnosed with conditions associated with moyamoya syndrome (MMS), including arteriosclerosis, autoimmune disease, meningitis, brain neoplasm, von Recklinghausen disease, Down syndrome, head trauma, irradiation to the head, among others ${ }^{1}$; or (2) the diagnostic text associated with MMD contained words such as 'uncertainty', 'undetermined', 'suspicious', '?' and other synonyms. The detailed process for case identification and ascertainment is described in online supplemental file 1 .

\section{Statistical analysis}

We estimated the national prevalence annually from 2013 to 2016. For the incidence, it is necessary to ascertain the first diagnosis of disease (ie, the disease onset). However, in the insurance data, there is no information about the disease history of an individual before the study start date. To handle this problem, a period before the index date (ie, wash-out period) is usually applied to judge the incident cases, which means only individuals free of target disease during the wash-out period are considered as incident cases. Thus, in this study, we calculated the national incidence in 2016 by using a 3-year wash-out period (2013-2015), in an attempt to minimise the possibility of misclassification of prevalent as incident MMD cases. ${ }^{9} 10$ Both rates were estimated using a two-stage approach. In the first stage, we calculated the incidence and prevalence in each province. The denominator $(N)$ was the total person years accrued in the UBMI-enrolled population in each province during the year. The numerator $(M)$ was the number of patients with MMD estimated from the denominator population in each province, considering missing data. The total enrolled population in each province was separated into three groups: individuals with no claim records (ie, those not using any medical service, $N_{0}$ ), individuals with complete diagnostic information in claim records $\left(N_{1}\right)$ and individuals with claim records but missing diagnostic information $\left(N_{2}\right)$. The patients $\left(M_{1}\right)$ with MMD that we observed directly were from $N_{1}$, but practically, a certain number of MMD patients $\left(M_{2}\right)$ existed in $N_{2}$. To minimise the impacts of missing data and determine the real rates, $M_{2}$ was estimated using a strategy based on Poisson regression with 10 times of multiple imputations (online supplemental file). In the second stage, considering the potential heterogeneity that may exist in data across provinces, we calculated the national or regional rates by pooling the provincespecific rates using a random-effects meta-analysis. The variance of the province-specific rates was stabilised with the Freeman-Tukey double arcsine transformation before pooling the data with meta-analysis model.

We estimated the incidence and prevalence overall and stratified by sex, age group and geographical region (East, North, Northeast, Northwest, Southcentral and Southwest). Rates were standardised using the population structure data in 2010, which was produced by China national census. All rates were reported as per 100000 person-years, and $95 \%$ CIs were calculated by assuming Poisson distribution. We performed sensitivity analyses to verify the robustness of the results: (1) excluding the top $10 \%$ or $20 \%$ of provinces with missing diagnostic information; (2) including observed cases only which were known as underestimation, to assess the lower bounds of the rates.

Costs for hospitalisation associated with MMD were calculated during 2013-2016, including total costs and medication costs per capita or per episode. Costs were discounted by Consumer Price Index (CPI) in each year to 2016 and converted into US dollars using the 2016 
Table 1 Characteristic of population enrolled in the urban basic medical insurance database in 22 provinces in urban China during 2013-2016

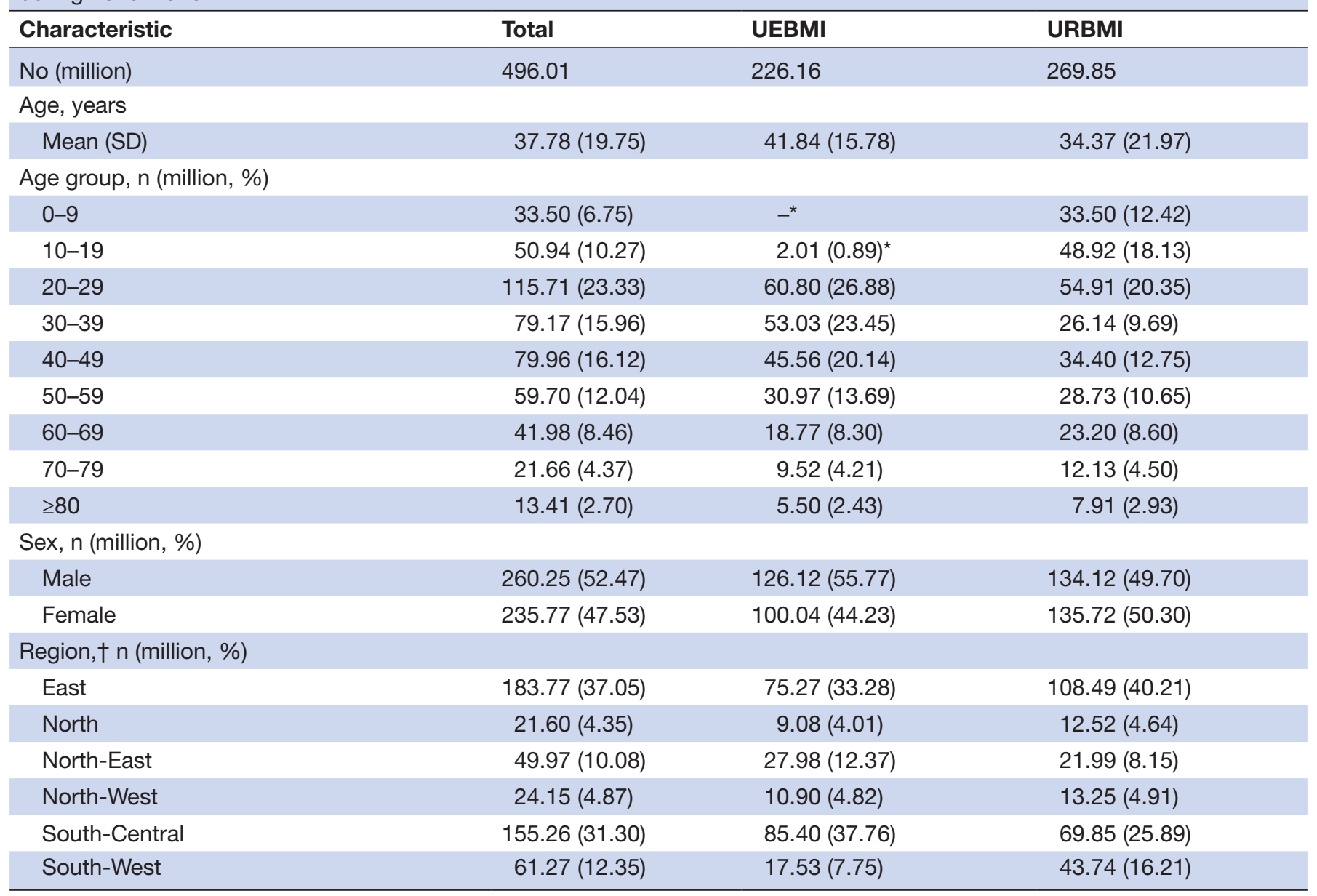

*UEBMI database covers urban working and retired employees (aged $\geq 18$ years old).

†East area included Jiangsu, Zhejiang, Anhui and Shandong provinces; North area included Shanxi and Inner Mongolia provinces; North-East area included Liaoning, Jilin and Heilongjiang provinces; North-West area included Shaanxi, Gansu, Qinghai and Xinjiang provinces; SouthCentral area included Henan, Hubei, Hunan, Guangdong, Guangxi and Hainan provinces; South-West area included Chongqing, Guizhou and Yunnan provinces.

UEBMI, Urban Employee Basic Medical Insurance; URBMI, Urban Residence Basic Medical Insurance.; .

RMB to US dollar exchange rate (period average). We also calculated the average number of hospital admissions and the average length of stay for inpatients.

We used the student's t-test for continuous variables and the $\chi^{2}$ test for categorical variables to compare characteristics between male and female patients. All statistical analyses were conducted with Stata V.14.0, and a two-sided test with $\mathrm{p}<0.05$ was determined statistically significant.

\section{RESULTS}

From 2013 to 2016 , a total of 496.01 million participants covered by the UEBMI and URBMI database were included (table 1), of whom 2168 were identified as patients with MMD. After excluding those with MMS $(\mathrm{n}=168)$ and those with suspicious MMD $(\mathrm{n}=13)$, a total of 1987 patients were confirmed as having MMD during the study period. Of all confirmed MMD patients, 988 were female, rendering a female-to-male ratio of 1.12 . The mean age of patients was $44.45 \pm 14.30$ years (table 2 ).

\section{Incidence}

The crude national incidence of MMD in 2016 was 0.59 (95\% CI 0.49 to 0.68 ). Incidence in females was 0.66 (95\% CI 0.51 to 0.81 ), higher than that in their male counterparts $(0.52,95 \%$ CI 0.40 to 0.65$)$ (figure 1$)$. The age-specific incidence showed a bimodal distribution, with the highest peak in middle-aged group and the second peak in child group. And the pattern was more prominent in females than in males (figure 1 and online supplemental table S1). Additionally, incidence varied by region, with a relatively higher rate in East China and South-central China (online supplemental table S1, S2).

The incidence standardised by 2010 China population census data was 0.42 per 100000 person-years (95\% CI 0.27 to 0.56 ), with 0.48 (95\% CI 0.32 to 0.64 ) in females and 0.36 (95\% CI, 0.23 to 0.49$)$ in males. In the sensitivity 
Table 2 Characteristic for patients with moyamoya disease in 22 provinces in urban China during 2013-2016



117 patients had missing data for age, 115 patients had missing data for gender.

*Student's t-test.

$+\chi^{2}$ test.

¥East area included Jiangsu, Zhejiang, Anhui and Shandong provinces; North area included Shanxi and Inner Mongolia provinces; North-East area included Liaoning, Jilin and Heilongjiang provinces; North-West area included Shaanxi, Gansu, Qinghai and Xinjiang provinces; SouthCentral area included Henan, Hubei, Hunan, Guangdong, Guangxi and Hainan provinces; South-West area included Chongqing, Guizhou and Yunnan provinces.

analysis, the results were similar to the rates reported above. The lower bound of the national incidence was $0.41(95 \%$ CI 0.40 to 0.43$)$ per 100000 person-years by considering only observed cases (online supplementary table S5).

\section{Prevalence}

The crude national prevalence of MMD in 2016 was 1.01 (95\% CI: 0.81 to 1.21 ) per 100000 person-years, reflecting a relatively rising trend from 0.62 (95\% CI 0.48 to 0.77$)$ per 100000 person-years in 2013 (online online supplementary table S3). Prevalence was higher in females (1.05, $95 \%$ CI: 0.83 to 1.27$)$ than in males $(0.90,95 \%$ CI: 0.70 to 1.09) (figure 2). Similar to the incidence, the age distribution of prevalence showed a bimodal pattern, and this pattern was more pronounced in females (figure 2 and online supplementary table S4). Moreover, East China had a relatively higher prevalence of MMD than other regions (online supplementary table S2, S4).

The standardised prevalence based on 2010 China population census data was 0.72 per 100000 person-years (95\% CI 0.50 to 0.95 ), with 0.80 (95\% CI 0.56 to 1.04 ) in females and 0.65 (95\% CI 0.44 to 0.85 ) in males. In the sensitivity analysis, we obtained broadly similar estimations and calculated the lower bound of the national prevalence of 0.67 (95\% CI 0.64 to 0.69$)$ per 100000 person-years in 2016 (online supplemental table S5).

Costs, number of admissions and length of stay for hospitalisation associated with MMD

From 2013 to 2016, 1262 patients $(63.51 \%)$ were hospitalised due to MMD (table 3). The average number of hospital admissions was 1.39 and the average length of stay was 18.69 days. The average inpatient cost per capita and 

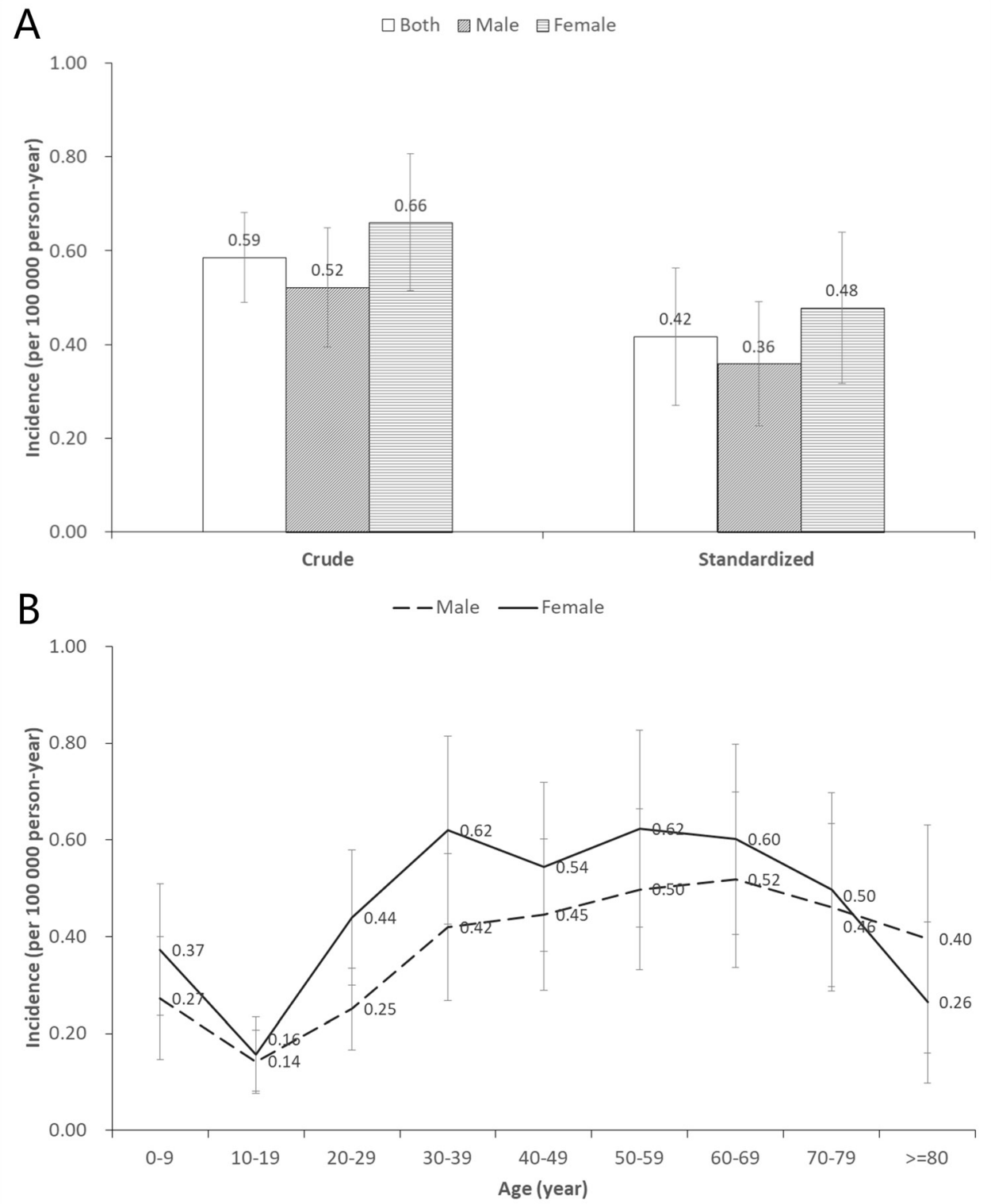

Figure 1 Incidence of moyamoya disease in urban China in 2016 (standardised by 2010 China census data). (A) Crude and standardised incidence by sex. (B) Crude incidence by sex and age.

per episode was US\$11 049 and US $\$ 7945$, respectively, of which about $40 \%$ was drug cost. The inpatient costs increased 2.03-fold from US\$4741 in 2013 to US\$9619 in 2016, showing a general upward trend.

\section{DISCUSSION}

In this nationwide population-based study, we calculated the incidence and prevalence of MMD in mainland China as 0.59 (95\% CI 0.49 to 0.68$)$ and 1.01 (95\% CI 0.81 to 1.21) per 100000 person-years, respectively. These estimates indicate that the rates of MMD in China were greater than those in the United States ${ }^{12}$ and Europe, ${ }^{14} 22$ but lower than recent values from other East Asian countries such as Japan ${ }^{7}$ and South Korea. ${ }^{910}$

Our results confirm that MMD is relatively common in East Asian populations, which may result from the distinct genetic background. Recent studies have demonstrated a significant association between the p.R4810K mutation in the ring finger protein 213 (RNF213) and $\mathrm{MMD},{ }^{23}$ and revealed a higher frequency of p.R4810K among East Asians compared with that in Caucasian populations. ${ }^{24-26}$ However, in different Asian countries, the effect sizes of p.R4810K were in great discrepancy. It significantly increased MMD risk in Japan and Korea and to a less degree in the Chinese population. ${ }^{24}$ Besides, MMD seems to have more complex determiners in China, and more than 40 other rare variants have also been identified. ${ }^{24} 27$ This may partly explain the lower MMD incidence in Chinese people compared with their Japanese and Korean counterparts. Additionally, a couple of socioeconomic or environmental factors could also play an important role in the regional difference in East Asia. 

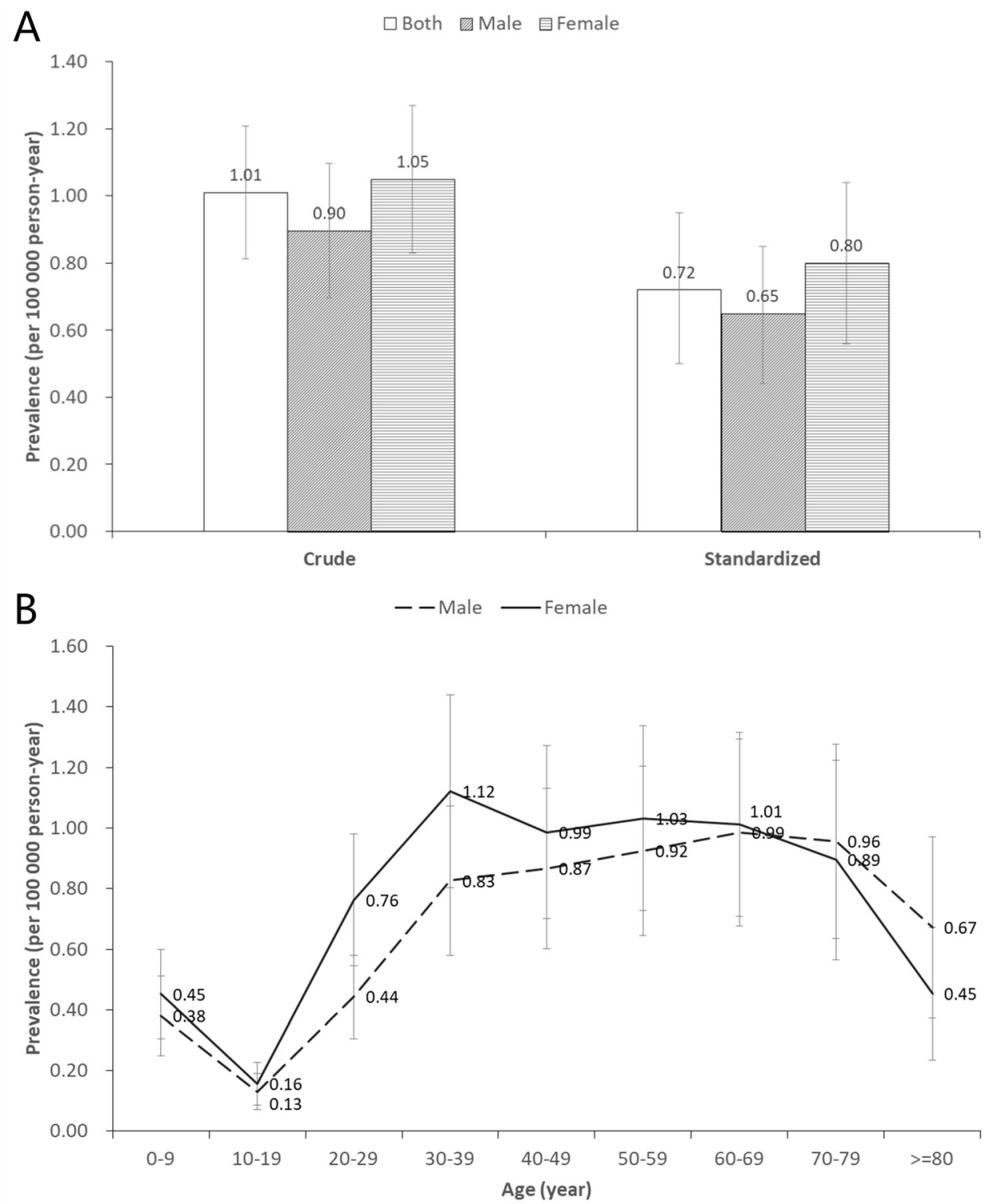

Figure 2 Prevalence of moyamoya disease in urban China in 2016 (standardised by 2010 China census data). (A) Crude and standardised prevalence by sex. (B) Crude prevalence by sex and age.

First, the accurate diagnosis of MMD relies on the findings from radiological techniques, such as brain CT, MRI and digital subtraction angiography (DSA). ${ }^{3}$ In Japan, appropriate diagnostic tools are widely available and a routine brain check-up system (the Brain Dock system) has been developed nationwide since it started in $1988 .{ }^{28}$ With the widespread use of this screening system, asymptomatic brain diseases could be identified and diagnosed in early stages ${ }^{29}$ as a consequence, the detection rate of MMD has increased. ${ }^{78} \mathrm{~A}$ similar phenomenon also existed in Korea ${ }^{30}$ However, a routine DSA and MRI check-up for a large population is impossible in China due to insufficient healthcare resources and limited financial capability. ${ }^{31} 32$ Some asymptomatic patients do not even realise the fact that they suffer from MMD, let alone be diagnosed with MMD. Second, underestimation of the actual incidence may occur in China due to a lack of awareness of MMD in neurologists as well as society. ${ }^{17}$ Chinese guidelines for the diagnosis and treatment of MMD were first issued in $2017 .^{33}$ At the time of this study, many neurologists, particularly those in undeveloped areas, knew little about MMD. This could also be proved by our findings that both the incidence and prevalence of MMD were the highest in East China, which has a higher economic level and more high-ranked tertiary hospitals than other regions. ${ }^{32}$ Besides, differences in other factors such as population structure, healthcare system and data collection method may also contribute to disparate rates of MMD by countries. Further research is required to confirm the reasons for the geographical variation.

MMD has been recognised as a disease that predominantly affects females, twice as much as males. ${ }^{34}$ Recent epidemiological studies reported female-to-male ratios ranging from 1.8 to 2.2 in Japan, ${ }^{5-7}$ and 1.8 to 1.9 in 


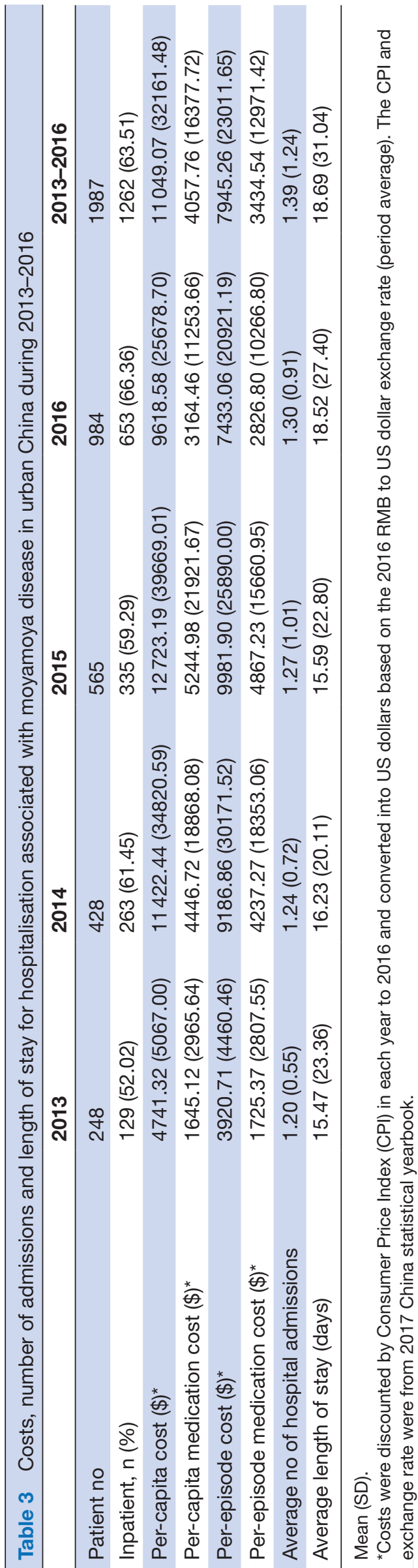

Korea. ${ }^{9} 10$ Meanwhile, in the USA and Europe, the female predominance is more pronounced with ratios ranging from 1.8 to $4.25 .^{12} 1435$ In the present study, we found that females had a higher incidence and prevalence of MMD than males in China, but the female predominance was relatively weak with a ratio of 1.12 , lower than that in other Asian and Western countries. This ratio was similar to the reports from Taiwan and Nanjing (a city in China), at 1.4 and 1.1, respectively. ${ }^{15} 16$ The observation of an ethnicity difference supports that genetic factors appear to play a major role in MMD. ${ }^{2317}$ More genetic analysis of Chinese patients with MMD might help to determine the pathogenesis of MMD in the future.

Another specific feature of MMD is the bimodal age distribution. The present study observed a two-peak pattern with the first peak in middle-aged group and the second peak in child group, consistent with previous studies in Asians, Caucasians, Hispanics and Africans. ${ }^{6-11}$ Furthermore, we found the peak in adults, particularly among females, was more prominent than in children. And the mean age of patients was 44.45 years old in our study, significantly higher than that in earlier Chinese studies before 2010 (about 25-35 years old). ${ }^{161819}$ These results may indicate that the highest peak of incidence of MMD appears to shift from children to adults. A similar pattern has been announced in Japan, Korea and Taiwan. ${ }^{81015}$ The increase of adult patients with MMD may associate with the increased prevalence of comorbid stroke and other neurological disorders. ${ }^{10} 1536$ Another explanation may be that some adult MMD patients presenting with stroke were previously misdiagnosed with arteriosclerosis or other diseases. ${ }^{17}$ However, the exact reasons for age differences remain unknown, and future studies are needed to find the causes.

We found more than half of the patients required hospitalisation due to MMD. The absolute number of admissions rose approximately fourfold from 2013 to 2016. This may indicate an actual increase of MMD cases. However, a more plausible explanation would be an increased detection of the disease due to recent advances in imaging techniques as well as its increased availability. ${ }^{15} 34$ Moreover, patients in China were more likely to have a longer hospital stay, an average of 18.69 days compared with 6.7 days for patients in the USA. ${ }^{37}$ The length of hospital stay is an important indicator to evaluate healthcare efficiency, as well as hospital resource utilisation. ${ }^{38}$ A three times longer hospital stay may suggest an inefficient diagnostic process and poor quality of medical care for MMD in China, although the length of stay might also be influenced by other factors, such as health insurance policy and patient severity. ${ }^{39}$ Prolonged length of stay also represents a significant economic problem on public health systems and their families. This study revealed costs per patient increased 2.03-fold from 2013 to 2016, reaching US $\$ 9619$. Although MMD is a rare disease, the disease burden is still heavy due to the high hospitalisation rate, long hospital stays and increasing costs. Clinicians and health policy-makers should explore effective 
ways to improve diagnosis and care for MMD patients in China.

This study used a large, nationally representative sample of the Chinese mainland population, estimating the incidence, prevalence and features for hospitalisation of MMD for the first time. It allowed us not only to provide an overall rate but also to explore age and sex patterns of rates across the country. It should be noted that our study, similar to many epidemiological studies of MMD, ${ }^{9}{ }^{15}$ was based on administrative data rather than using a registry design. We must acknowledge that each data source has its own advantages and limitations: registry studies are effective for case ascertainment but less feasible for studying rare conditions due to the difficulty of ensuring a large population. ${ }^{40}$ Alternatively, claims data can ensure a large sample for studies on rare diseases, but may not provide more detailed information for each case. Therefore, this study has several limitations. First, missing values were an unavoidable problem in research based on administrative databases, which would affect the estimates if we ignored it. However, we adopted an imputation strategy and conducted sensitivity analyses to explore the potential influence on the estimations. ${ }^{40}$ Second, the lack of detailed information, such as clinical symptoms, laboratory data and imaging results in the insurance claims database, precluded the possibility to identify disease type and to describe the genetic features. We were also unable to contact patients directly to obtain additional information because of the anonymity requirement. Third, research using electronic medical databases is reliant on the accuracy of diagnosis. We validated the diagnosis of MMD in the UEBMI and URBMI databases by reviewing individual patient charts in one hospital (online supplemental file). The positive predictive value (PPV) was $87 \%$, quite comparable to a Danish population-based study on MMD (with a PPV of $86 \%$ ). ${ }^{14}$ Nevertheless, some extent of misclassification of MMD cases might still occur in the database. Forth, we set a 3-year wash-out period to define the incident cases, which may be not sufficient, potentially affecting the estimation of incidence. Fifth, patients may be missed in claim data if they visited private institutions where insurance cards cannot be used. But the proportion of this population should be minor. Sixth, retrospective studies based on electronic medical databases may be affected by differences in clinical practice and medical level between clinicians and hospitals as well as changes over time. Finally, the database did not cover rural inhabitants and certain urban populations such as military soldiers, which have a different insurance system. The exclusion of these groups may have affected the estimations.

\section{CONCLUSION}

This research fills a gap in the incidence and prevalence of MMD in mainland China. Our results confirm that MMD is relatively common in East Asian populations, but the rates in China were lower than those in other East
Asian countries such as Japan and South Korea. We recognised the higher incidence and prevalence in females compared with males and the bimodal age distribution, as previously reported. And some unique epidemiologic features, including a relatively weak female predominance and a shift in the highest peak of incidence from children to adults, revealed new sight into MMD. Although MMD is a rare disease, the disease burden is relatively heavy for patients due to the high hospitalisation rate, long hospital stays and increasing costs. Further research is warranted to examine the potential pathogenesis and appropriate therapeutic strategies, in order to manage this progressive cerebrovascular disease more cost-effectively.

\section{Author affiliations}

${ }^{1}$ Department of Epidemiology and Biostatistics, School of Public Health, Peking University Health Science Centre, Beijing, China

${ }^{2}$ Department of Geriatric Neurology, Qilu Hospital of Shandong University, Jinan, China

${ }^{3}$ Department of Neurology, Peking University Third Hospital, Beijing, China ${ }^{4}$ Peking University Health Information Technology Co. Ltd, Beijing, China ${ }^{5}$ Beijing Healthcom Data Technology Co. Ltd, Beijing, China

${ }^{6}$ Department of Medical Affairs, Qilu Hospital of Shandong University, Jinan, China

Contributors Concept and design: YS, GZ, PG, SW and SZ. Drafting of the manuscript: YS, GZ and SW. Critical revision of the manuscript for important intellectual content: SW and SZ. Provision of study material or patients: JW, YS and QW. Collection and assembly of data: JW, GL, YS, JF, JY, XY, ZY and QW. Check and approve of clinical definition: GZ and LC. Data analysis: YS and JF. Data interpretation: GZ, YS, PG, SW, SZ and QW. Administrative, technical or material support: PG, SW and SZ. Supervision: SW and SZ. Final approval of manuscript: all authors.

Funding This work was supported by the National Natural Science Foundation of China (grants 91646107, 81973146 and 81502884) and Peking University Medicine Seed Fund for Interdisciplinary Research: The Fundamental Research Funds for the Central Universities (BMU2020MX017).

Disclaimer The funders had no role in study design, data collection and analysis, decision to publish, or preparation of the manuscript.

Competing interests None declared.

Patient consent for publication Not required.

Ethics approval The study protocol was approved by the ethical review committee of the Peking University Health Science Center (IRB. No: IRB00001052-18012). Provenance and peer review Not commissioned; externally peer reviewed. Data availability statement Data may be obtained from a third party and are not publicly available. The data that support the findings of this study are available from National Healthcare Security Administration of China but restrictions apply to the availability of these data, which were used under license for the current study, and so are not publicly available. Data are however available from the authors upon reasonable request and with permission of National Healthcare Security Administration of China.

Open access This is an open access article distributed in accordance with the Creative Commons Attribution Non Commercial (CC BY-NC 4.0) license, which permits others to distribute, remix, adapt, build upon this work non-commercially, and license their derivative works on different terms, provided the original work is properly cited, appropriate credit is given, any changes made indicated, and the use is non-commercial. See: http://creativecommons.org/licenses/by-nc/4.0/.

ORCID iD

Yixin Sun http://orcid.org/0000-0002-4879-0529

\section{REFERENCES}

1 Kuroda S, Houkin K. Moyamoya disease: current concepts and future perspectives. Lancet Neurol 2008;7:1056-66. 
2 Scott RM, Smith ER. Moyamoya disease and moyamoya syndrome. N Engl J Med 2009;360:1226-37.

3 Shang S, Zhou D, Ya J, et al. Progress in moyamoya disease. Neurosurg Rev 2020;43:371-82.

4 Kleinloog R, Regli L, Rinkel GJE, et al. Regional differences in incidence and patient characteristics of moyamoya disease: a systematic review. J Neurol Neurosurg Psychiatry 2012;83:531-6.

5 Kuriyama S, Kusaka Y, Fujimura M, et al. Prevalence and clinicoepidemiological features of moyamoya disease in Japan: findings from a nationwide epidemiological survey. Stroke 2008;39:42-7.

6 Sato Y, Kazumata K, Nakatani E, et al. Characteristics of moyamoya disease based on national registry data in Japan. Stroke 2019;50:1973-80.

7 Hayashi K, Horie N, Suyama K, et al. An epidemiological survey of moyamoya disease, unilateral moyamoya disease and quasi-moyamoya disease in Japan. Clin Neurol Neurosurg 2013;115:930-3.

8 Baba T, Houkin K, Kuroda S. Novel epidemiological features of moyamoya disease. J Neurol Neurosurg Psychiatry 2008;79:900-4.

9 Ahn IM, Park D-H, Hann HJ, et al. Incidence, prevalence, and survival of moyamoya disease in Korea: a nationwide, population-based study. Stroke 2014;45:1090-5.

$10 \mathrm{Kim}$ T, Lee H, Bang JS, et al. Epidemiology of moyamoya disease in Korea: based on national health insurance service data. $J$ Korean Neurosurg Soc 2015;57:390-5.

11 Uchino K, Johnston SC, Becker KJ, et al. Moyamoya disease in Washington state and California. Neurology 2005;65:956-8.

12 Ghaffari-Rafi A, Ghaffari-Rafi S, Leon-Rojas J. Socioeconomic and demographic disparities of moyamoya disease in the United States. Clin Neurol Neurosurg 2020;192:105719.

13 Saarela M, Mustanoja S, Pekkola J, et al. Moyamoya vasculopathy Patient demographics and characteristics in the Finnish population. Int J Stroke 2017;12:90-5.

14 Birkeland P, Tharmabalan V, Lauritsen J, et al. Moyamoya disease in a European setting: a Danish population-based study. Eur J Neurol 2020;27:2446-52.

15 Chen P-C, Yang S-H, Chien K-L, et al. Epidemiology of moyamoya disease in Taiwan: a nationwide population-based study. Stroke 2014;45:1258-63.

16 Miao W, Zhao P-L, Zhang Y-S, et al. Epidemiological and clinical features of moyamoya disease in Nanjing, China. Clin Neurol Neurosurg 2010;112:199-203.

17 Bao X-Y, Wang Q-N, Zhang Y, et al. Epidemiology of moyamoya disease in China: single-center, population-based study. World Neurosurg 2019;122:e917-23.

18 Duan L, Bao X-Y, Yang W-Z, et al. Moyamoya disease in China: its clinical features and outcomes. Stroke 2012;43:56-60.

19 Liu X-ju, Zhang D, Wang S, et al. Clinical features and long-term outcomes of moyamoya disease: a single-center experience with 528 cases in China. J Neurosurg 2015;122:392-9.

20 Tian Y, Liu H, Si Y, et al. Association between temperature variability and daily hospital admissions for cause-specific cardiovascular disease in urban China: a national time-series study. PLoS Med 2019;16:e1002738.

21 Xiong X, Zhang Z, Ren J, et al. Impact of universal medical insurance system on the accessibility of medical service supply and affordability of patients in China. PLoS One 2018;13:e0193273.
22 Doherty RJ, Caird J, Crimmins D, et al. Moyamoya disease and moyamoya syndrome in Ireland: patient demographics, mode of presentation and outcomes of EC-IC bypass surgery. Ir J Med Sci 2021:190:335-44.

23 Kamada F, Aoki Y, Narisawa A, et al. A genome-wide association study identifies RNF213 as the first moyamoya disease gene. J Hum Genet 2011;56:34-40.

24 Liao X, Deng J, Dai W, et al. Rare variants of Rnf213 and moyamoya/ non-moyamoya intracranial artery stenosis/occlusion disease risk: a meta-analysis and systematic review. Environ Health Prev Med 2017;22:75

25 Cecchi AC, Guo D, Ren Z, et al. Rnf213 rare variants in an ethnically diverse population with moyamoya disease. Stroke 2014;45:3200-7.

26 Liu W, Senevirathna STMLD, Hitomi T, et al. Genomewide association study identifies no major founder variant in Caucasian moyamoya disease. J Genet 2013;92:605-9.

27 Zhang Q, Liu Y, Zhang D, et al. Rnf213 as the major susceptibility gene for Chinese patients with moyamoya disease and its clinical relevance. J Neurosurg 2017;126:1106-13.

28 Morita A. Value of brain DOCK (brain screening) system in Japan. World Neurosurg 2019;127:502.

29 Ikeda K, Iwasaki Y, Kashihara H, et al. Adult moyamoya disease in the asymptomatic Japanese population. $J$ Clin Neurosci 2006;13:334-8

30 Lee SU, Kim T, Kwon O-K, et al. Trends in the incidence and treatment of cerebrovascular diseases in Korea : Part II. cerebral infarction, cerebral arterial stenosis, and moyamoya disease. $J$ Korean Neurosurg Soc 2020;63:69-79.

31 Liu Y, Rao K, Wu J, et al. China's health system performance. Lancet 2008;372:1914-23.

$32 \mathrm{Yu} \mathrm{M}, \mathrm{He} \mathrm{S}$, Wu D, et al. Examining the multi-scalar unevenness of high-quality healthcare resources distribution in China. Int J Environ Res Public Health 2019;16. doi:10.3390/ijerph16162813. [Epub ahead of print: 0708 2019].

33 Chinese expert group on diagnosis and treatment of moyamoya disease and moyamoya syndrome. Guidelines for the diagnosis and treatment of moyamoya disease and moyamoya syndrome in China (2017). Chin J Neurosurg 2017;33:541-7.

34 Xiaowen S, Qian Z, Jizong Z. Research progress in the epidemiology of moyamoya disease. Chin J Cerebrovasc Dis 2021;15:9-15.

35 Kraemer M, Heienbrok W, Berlit P. Moyamoya disease in Europeans. Stroke 2008;39:3193-200.

36 Wang $\mathrm{W}$, Jiang B, Sun $\mathrm{H}$, et al. Prevalence, incidence, and mortality of stroke in China: results from a nationwide population-based survey of 480687 adults. Circulation 2017;135:759-71.

37 Kainth D, Chaudhry SA, Kainth H, et al. Epidemiological and clinical features of moyamoya disease in the USA. Neuroepidemiology 2013:40:282-7.

38 Marfil-Garza BA, Belaunzarán-Zamudio PF, Gulias-Herrero A, et al. Risk factors associated with prolonged hospital length-of-stay: 18year retrospective study of hospitalizations in a tertiary healthcare center in Mexico. PLoS One 2018;13:e0207203.

39 Schouten LMT, Hulscher MEJL, Akkermans R, et al. Factors that influence the stroke care team's effectiveness in reducing the length of hospital stay. Stroke 2008;39:2515-21.

$40 \mathrm{Xu} \mathrm{L}$, Chen L, Wang S, et al. Incidence and prevalence of amyotrophic lateral sclerosis in urban China: a national populationbased study. J Neurol Neurosurg Psychiatry 2020;91:520-5. 